\title{
EDITORIAL
}

\section{Special collection on mobile mixed reality 2019 update}

Thomas Cochrane ${ }^{\mathrm{a} *}$, Vickel Narayan ${ }^{\mathrm{b}}$ and James Birt

${ }^{a}$ altLAB, Auckland University of Technology, Auckland, New Zealand; ${ }^{b}$ University of Sydney, Camperdown, NSW, Australia; 'Faculty of Society \& Design Bond University, Gold Coast, QLD 4229, Australia

This special collection of Research in Learning Technology explores the development of the state of the art of Mobile Mixed Reality (MMR) in education. The special collection was established in 2018 to provide research-informed exploration of this emergent and rapidly developing arena of educational technology through the lens of Scholarship Of Technology Enhanced Learning (SOTEL). The special collection update for 2019 includes four articles that cover self-efficacy and motivation of MMR users, analysis of student experiences of MMR, and a selection of case studies on designing and implementing MMR in educational contexts. The range of articles illustrates the further development of MMR as a platform for designing authentic learning environments in both formal and informal learning situations. The articles also highlight attempts to address the issue identified in the 2018 collection of a general lack of engagement with new learning theories and models in the use of MMR to design transformative learning experiences.

Keywords: design-based research; scholarship of technology enhanced learning; augmented reality; virtual reality; mixed reality; mobile learning

This editorial is part of the special collection 'Mobile Mixed Reality-Enhanced Learning' edited by Thomas Cochrane, Vickel Narayan, James Birt, Helen Farley and Fiona Smart. Read all articles from this collection here.

\section{Introduction}

In this special collection, we explore the design of educational experiences that integrate Mobile Mixed Reality (MMR) technologies into both formal and informal learning environments. We are particularly interested in applications of MMR in education that are informed by scholarly educational design approaches (Haynes 2016) such as design-based research or DBR, also termed as educational design research or EDR (McKenney and Reeves 2019). DBR has emerged as a highly suitable methodology for the iterative design, implementation and evaluation of MMR (Bannan, Cook, and Pachler 2015). While there have been many novel and often technocentric applications of MMR in education, four key areas have emerged as particularly relevant for the authentic application of MMR learning environments: simulation - particularly clinical health simulation (Pottle 2019), automation, cost-prohibitive learning experiences (e.g. replacing class field trips with virtual tours), and high-risk learning environments (Cochrane, Smart, and Narayan 2018). 
MMR also provides a foundation for the designing of self-regulated learning environments (Blaschke and Hase 2019).

\section{MMR in education}

The special collection update attempts to provide an answer to the following question: Where has the research and practice of MMR in education got to in 2019? The authors of the four published articles explore some of the emergent examples of the design of MMR learning environments.

\section{In this issue}

Here we explore the emergent themes drawn from the four articles included in this special collection update for 2019.

\section{Exploring mixed reality based on self-efficacy and motivation of users}

In this article, Essmiller et al. (2020), the authors, gathered quantitative data from 63 college students to investigate how the effects of cognitive load could be negated in a mixed reality-based learning environment. They state that a learner experiences significant cognitive load while engaging in a virtual environment wearing a headmounted display (HMD), such as the Microsoft HoloLens - impacting on the learning experience and outcome. To test this hypothesis, they devised their study using learner motivation and self-efficacy as indicators while engaging students in three different virtual reality (VR) learning experiences: (1) Roboroid, (2) Tutorial and (3) Freeplay using the Microsoft HoloLens. The 63 students were randomly assigned to each learning activity - the students were not told what activity they were going to engage with. The students completed the survey immediately after completing the learning activity in VR environment. The survey data were analysed using oneway analysis of variance (ANOVA) using the SPSS Data Editor. From the analysis, the authors found that there were no significant differences between the two factors across all three VR learning types - suggesting cognitive load was a non-issue for learners. They comment that the students perhaps learn to manage their cognitive load after the initial exposure to VR environment by gradually learning the skills and competencies needed to navigate and learn in virtual space. They also found that Roboroid and Freeplay types of learning activities in mixed VR enhanced students' motivation, while learning similar to a tutorial encouraged self-efficacy. They conclude the study by stating that mixed VR offers significant opportunities for facilitating learning; however, further research is needed to explore the attributes of a constructive and effective mixed VR design.

\section{MESH360: a framework for designing MMR-enhanced clinical simulations}

Cochrane et al. (2020), the authors, report on their findings from a design-based research project of designing and implementing an MMR virtual environment for use in paramedicine. In an attempt to design and investigate an authentic MMR learning environment, the authors derived a set of guiding principles from the literature. They discuss five principles in their article and how these are used as 
a guide for designing the learning intervention, which was evaluated and refined over a 2-year period. They share their learning designs, creative and authentic strategies for developing and embedding MMR in learning and teaching and innovative data collection strategies to measure the authenticity of the design. Over the 2 years of the project, several sets of qualitative data were collected from different cohorts of students and professional paramedic practitioners. These data were triangulated and thematically analysed to address the following overarching research question of the study: How can we design clinical simulation learning environments that are more authentic (than the current practice), facilitate the development of higher-order critical thinking and are cost-effective? The authors using triangulated participant data, including biometric data, conclude that pedagogical design and embedded use of MMR for facilitating clinical simulation-based training enhances the learning experience by amplifying the authenticity of the tasks and activities the students engage in. This requires the students to reflect and progress the information and their own experience in the MMR environment at a deeper level - triggering higher cognitive processes. As a significant outcome of the project, the authors provide a set of refined design principles or transferrable knowledge that could be used as a guide by practitioners exploring MMR for use in clinical training and education.

\section{Analysing construction students' experiences of MMR-enhanced learning in VR and augmented reality environments}

Vasilevski and Birt (2020) in their article investigate the use of MMR in construction education, in particular, for improving the learning experience and outcome. They developed and investigated how MMR technology and design strategies could be used to allow students to build an understanding and application of the new building information modelling (BIM) workflows in a postgraduate construction course. As a two-part learning experience, a non-traditional lecture was delivered to the students. The teachers provided a guided tour of a virtual environment using MMR, as it was being used in the industry for applying and understanding BIM. In the second part of the learning experience, the students in groups got a hands-on experience of actively exploring and investigating VR and augmented reality (AR) environments of Building Information Management (BIM) application in the industry. Qualitative data from both learning experiences were collected from two cohorts of 45 students over two semesters (a total of 90 students participated in the study). The students were required to submit a reflective essay on their learning experience of both the activities - analysed to draw themes and categories to inform the findings of the study. The emergent themes were grouped into three categories (1) VR experience, (2) AR experience and (3) learning environment, and the authors have discussed their implications on the learning experience, the learner and MMR in more detail in the article. Using their analysis, the authors address the following research question: 'Does applied mobile mixed reality create an enhanced learning environment for students?' They state yes, it does and discuss several factors that are enhanced or contribute to a positive experience in an MMR learning environment, such as improved learning engagement, immersion and motivation, improved interaction, and increased fun and enjoyment. They highlight that while the study has provided some glimpses into what motivates and excites students in an MMR environment, further research is needed to understand and identify features and unique opportunities that are still emergent. 


\section{T. Cochrane et al.}

\section{A framework for mixed reality free-choice self-determined learning}

Aguayo, Eames and Cochrane (2020) in their article explore MMR as a platform for enabling self-determined learning to enhance ecological literacy in free-choice educational settings, such as museums and visitor centres. They too used a design-based research approach to design and develop an MMR-based learning intervention to facilitate student-determined learning for enhancing ecological literacy. Using key pedagogical frameworks, such as heutagogy, bring-your-own-device (BYOD) and self-determined learning - frameworks pertinent to their research and research aims, they created a set of design principles to guide the development of the learning intervention. The authors discuss the design, development, evaluation and refinement of intervention using the four-phase analytical framework and activity theory model. The authors discuss the main tasks and activities they completed in the first phase mainly consultative data gathering and planning work. In the second phase the design of the learning environment using Google Communities for establishing the network is discussed; the design of MMR learning intervention is discussed in the third phase, and the final phase discusses the implementation of the intervention and data gathering for evaluation. Qualitative data from the students were collected and analysed using the socio-cultural activity theory analytical framework. The authors address the research question, 'How can mobile learning be designed with EOTC (education outside the classroom) to enhance the ecological literacy of students and their parents?', by providing a comprehensive list of design principles and a thick description of how they were integrated into the development of intervention. The design was evaluated using the data that helped understand the impact of students. The authors report that the MMR environment that was designed to help build ecological literacy of students 'appeared to heighten student engagement and learning', promoted 'postvisit' learning and 'assisted some knowledge and attitude development'. They noted that 'the activities that engaged students the most are located at either end of the MR immersion continuum, leading to speculation that the continuum might actually exist as a circle rather than a line'. The authors outline several findings from the study as discussed in depth in the article.

\section{Thoughts on the future of MMR}

It is certainly easy to conclude that technology enhances learning, and indeed the upcoming Bring Your Own Device (BYOD) smartphone technologies will capture the creative imagination of MMR education delivery, including, the shift towards $5 \mathrm{G}$ wireless networking, depth sensor cameras, integrated LiDAR and more complex machine learning algorithms capable of data processing.

However, predicting the future is difficult and made more complex with the rapid rise in technology innovation. Recently, we have seen changes to the support of mobile VR with a shift away from mobile phone insertable HMDs (Google Cardboard, Samsung Gear VR) and 3-DoF headsets (Oculus Go) to 6-DoF standalone hardware (Oculus Quest, HTC VIVE Focus). Software support has also changed recently with companies such as Unity3D depreciating native support for various VR and AR hardware from their game engine requiring third party plug-ins.

If the COVID-19 pandemic has highlighted anything, it is that there is a greaterthan-ever need for communicative technology in education, but even more so a willingness of educators and learning providers to understand, support and manage mobile 
technology-enhanced learning. The future of MMR will not be driven through technology innovation alone as discussed in this special issue. Rather it will be a multi-faceted approach through understanding technology innovation, managing changes, integrating learner-centred design methodology, educator literacy and increased capacity for learning providers to support the scalable deployment of MMR devices and applications. It is therefore these current and the near future challenges that we look forward to exploring in the 2020 edition of the special collection on MMR.

\section{Acknowledgements}

The special issue guest editors would like to express their thanks to the RLT editorial team for allowing them to explore this special issue on MMR. Special gratitude goes to the team of reviewers and authors for getting the special issue through in such a short time frame.

\section{References}

Aguayo, C., Eames, C. \& Cochrane, T. (2020) 'A framework for mixed reality free-choice, self-determined learning', Research in Learning Technology, vol. 28. doi: 10.25304/rlt.v28.2347

Bannan, B., Cook, J. \& Pachler, N. (2015) 'Reconceptualizing design research in the age of mobile learning', Interactive Learning Environments, vol. 24, pp. 1-16. doi: $10.1080 / 10494820.2015 .1018911$

Blaschke, L. M. \& Hase, S. (2019) 'Heutagogy and digital media networks: setting students on the path to lifelong learning', Pacific Journal of Technology Enhanced Learning, vol. 1, pp. 1-14. doi: 10.24135/pjtel.v1i1.1

Cochrane, T., et al., (2020) 'MESH360: a framework for designing MMR-enhanced clinical simulations', Research in Learning Technology, vol. 28. doi: 10.25304/rlt.v28.2357

Cochrane, T., Smart, F. \& Narayan, V. (2018) 'Editorial for RLT special issue on mobile mixed reality', Research in Learning Technology, vol. 26, p. 2195. doi: 10.25304/rlt.v26.2195

Essmiller, K., et al., (2020) 'Exploring mixed reality based on self-efficacy and motivation of users', Research in Learning Technology, vol. 28. doi: 10.25304/rlt.v28.2331

Haynes, D. (2016) 'Introducing SOTEL', International Journal for the Scholarship of Technology Enhanced Learning, vol. 1, pp. 1-2. [online] Available at: http://ejournals.library.gatech.edu/ ijsotel/index.php/ijsotel/article/view/17

Mckenney, S. \& Reeves, T. (2019) Conducting Educational Design Research, Routledge, London. doi: $10.4324 / 9781315105642$

Pottle, J. (2019) 'Virtual reality and the transformation of medical education', Future Healthcare Journal, vol. 6, p. 181. doi: 10.7861/fhj.2019-0036

Vasilevski, N. \& Birt, J. (2020) 'Analysing construction student experiences of mobile mixed reality enhanced learning in virtual and augmented reality environments', Research in Learning Technology, vol. 28. doi: 10.25304/rlt.v28.2329 